\title{
Spectrum Handoff Strategies for Multiple Channels Cognitive Radio Network
}

\author{
Adisorn Lertsinsrubtavee, Naceur Malouch, and Serge Fdida \\ Université Pierre et Marie Curie - Paris 6 (UPMC-LIP6) \\ \{Adisorn.Lertsinsrubtavee,Naceur.Malouch,Serge.Fdida\}@lip6.fr
}

\begin{abstract}
This paper proposes a new spectrum handoff approach for multiple channels cognitive radio networks to support delay sensitive applications such as VoIP. The delay probability is estimated to determine whether and how to perform the handoff operation. Moreover, traffic prediction on licensed channels is also required in order to estimate the impairment in the delay. Since, the error of prediction still cannot be solved totally, therefore the backup channel solution is proposed to reduce the harmfulness of this impact. The delay probability density function is through various strategies of spectrum handoff for multiple channels cognitive radio.
\end{abstract}

\section{INTRODUCTION}

Recently, the shortage of licensed spectrum is becoming the critical issue for the new emerging wireless communication services. The limited available spectrum and inefficiency in spectrum usage necessitate a new communication paradigm to exploit the existing spectrum opportunistically [1]. However, the FCC Spectrum Policy Task Force [2] reported a utilization of allocated spectrum varying from $15 \%$ to $85 \%$ in the band below $3 \mathrm{GHz}$. The significant under utilization has inspired many research works to pay efforts to the Cognitive Radio (CR) technology. CR networks can improve the spectrum utilization by allowing CR users temporarily occupying the licensed channels when they are unused by licensed users. The capabilities of CR technology can be classified into four main functionalities including spectrum sensing, spectrum decision, spectrum sharing, and spectrum mobility or spectrum handoff. Most of the existing research works concentrate on spectrum sensing, spectrum sharing and spectrum decision, but a few works on spectrum handoff have been done for cognitive radio networks espe-

Permission to make digital or hard copies of all or part of this work for personal or classroom use is granted without fee provided that copies are not made or distributed for profit or commercial advantage and that copies bear this notice and the full citation on the first page. To copy otherwise, to republish, to post on servers or to redistribute to lists, requires prior specific permission and/or a fee.

ACM CoNEXT 2010, November 30 - December 3 2010, Philadelphia, USA.

Copyright 2010 ACM 1-4503-0448-1/10/11 ...\$10.00. cially in the case when transmissions are done over multiple channels in parallel.

The spectrum handoff occurs when licensed users are detected in the channel. Thus, CR user has to leave the channel immediately and search for a new available one. During the handoff operation, the communication of CR user has to be interrupted and packets must wait in the transmission buffer. The communication can be resumed when the connection is successfully moved to the new channel. From this point of view, large waiting delays can be incurred and packets may be lost. Sometimes, licensed users may use the channel only for short periods intermittently. The frequency of spectrum handoff can be reduced if CR users wait until licensed users finish their transmission to send again over the same channel. The studies [3] and [4] have mentioned such possibility. Here, predicting the behavior of licensed users in the near future assists CR users to decide whether it should keep the current channel or leave it. Typically, prediction should improve the performance of the spectrum handoff [5].

Nevertheless, the error of prediction cannot be avoided and hence impacts the evaluation of channel quality. We propose the use of backup channels as a compromised solution to alleviate this problem. The CR users can reserve temporarily additional channels at the same time with the main new channel. In case the behavior of licensed users is different than expected, the backup channels can serve the communication immediately without extra preparation time. Besides, the spectrum handoff procedure is further complicated since the cognitive radio usually handle multiple channels for transmission. Thus, it is not obvious to determine which channels to select. We identify several strategies when handoff must be performed.

\section{MULTIPLE CHANNELS HANDOFF}

We consider the end-to-end delay as the most important quality factor. Our approach is then based on estimating the delay probability density function (pdf) continuously by emulating a transmission over all licensed channels, even those which are not used for the current transmission. A channel can be modeled as an ON-OFF pattern (Fig.1). Prediction model allows CR users to predict the next OFF period on previous traffic patterns. The CR user can estimate the pdf at 
the end of next OFF period along with the past computation. Each packet delay obtained from the past and the prediction is recorded and compared with a maximum satisfactory delay (MaxD). Therefore, the probability that the delay is larger than MaxD is computed. This probability must stay below some small threshold.

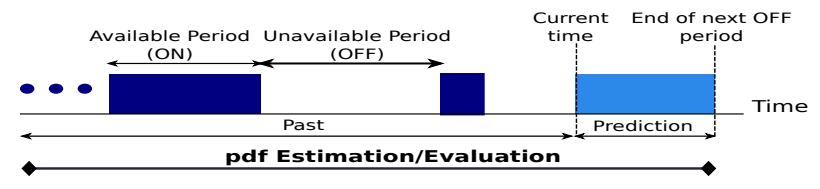

Figure 1: The delay probability function estimation

Since the channel selection is based on prediction and past pdf, the handoff procedure may select an inadequate channel. Unfortunately, this error of prediction still cannot be eliminated. The delay can be then increased until over the threshold and CR users will need to perform handoff again. Using a backup channel can reduce the damage of this error by allowing CR users reserving temporarily a channel in addition to the new one selected by the handoff procedure. When an error occurs, the communication can be switched to this backup channel immediately without any preparation time. Fig.2 shows the classification of different spectrum handoff approaches for multiple channels cognitive radio depending on the used evaluation strategy and the possible activation of the backup channel. Remind that CR user is possibly sending over multiple active channels in parallel.

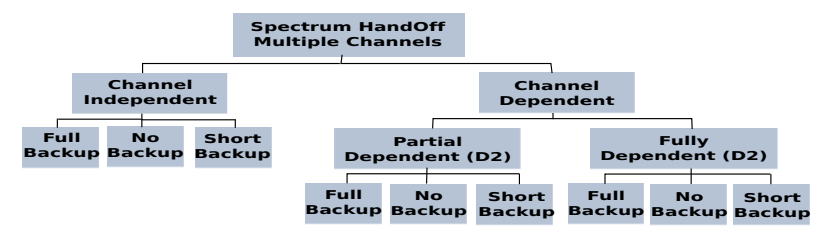

Figure 2: Spectrum handoff classification for multiple channel cognitive radio

Channel independent:Spectrum handoff is performed for each channel individually regardless of the state of other transmissions over the other channels maintained by CR user.

Partial Channel dependent (D1):Licensed users are detected in one of the multiple channels and need to perform handoff. The CR user chooses a new available channel by evaluating its quality combined with the existing active channels. The channels which has the best performance from the global evaluation will be selected to be a new active channel. For example, a CR user is using channel number 1 to 4. Once, the licensed users are detected on channel number 4 , and channel number 5 and 6 are available. The global evaluation will be performed by set of channels $(1,2,3,5)$ and $(1,2,3,6)$. If the result turned out that the group $(1,2,3,6)$ had better performance, the CR users would select the channel number 6 to be a new active channel.

Fully Channel dependent (D2): In this case the global evaluation will be performed on all possible combinations of all available channels. Regarding the previous example, if the channel number 4 becomes unavailable, channel number 1,2,3,5,6 will be re-evaluated. The existing available channels $(1,2,3)$ might not be involved in the new set of multiple channels. If the result of global evaluation turned out that the combination $(2,3,5,6)$ has the best performance, the channel number 1 and 4 would be performing handoff operation.

Backup channel solutions: CR users will keep a backup channel in order to prevent the degradation of quality due to prediction errors. In addition, when one of the active channels needs to perform handoff, it can be switched to this backup channel immediately without any preparation time or delay. There are two possibilities of backup solution for our approach. First is the full backup solution where CR users keep at least one backup all the time. Although the full backup can reduce significantly the impact of prediction errors, the spectrum utilization is highly decreased. The Short Time Backup Channel (STBC) approach is a compromised solution between full backup and no backup. CR users keep the STBC for a short period and release it when the active channels become confident. For example, after the handoff operation, the delay probability can be high because there are many packets in the queue. But, if after some time, this probability is reduced under the threshold, the STBC can be released. Otherwise, it will be used and it is the first channel that is released.

\section{SUMMARY AND CURRENT WORK}

This paper has proposed a several spectrum handoff approaches for multiple channels cognitive radio networks in order to support delay sensitive applications.CR users calculate the delay probability density function and decide whether to perform a handoff operation. Furthermore, we introduce a novel backup channel technique to reduce the impact of error prediction on the handoff operation. Currently, we are investigating the multiple channel strategies with backup channel through several scenarios. The STBC is expected to be a good tradeoff if channels are released at the right time.

\section{REFERENCES}

[1] I. F. Akylidiz, W. Y. Lee, M. C. Vuran, and S. Mohanty, "Next generation/dynamic spectrum access/cognitive radio wireless networks: a survey," Computer Networks, vol. 50, no. 13, pp. 2127-2159, 2006.

[2] "ET Docket 03-222 Notice of proposed rule making and order." FCC, Dec 2003.

[3] S. Talat and L.-C. Wang, "Qos-guaranteed channel selection scheme for cognitive radio networks variable channel bandwidths," in Proc. IEEE ICCCAS, 2009.

[4] C.-W. Wang and L.-C. Wang, "Modeling and analysis for proactive-decision spectrum handoff in cognitive radio networks," in Proc. IEEE ICC, 2009.

[5] M. Hoyhtya, S. Pollin, and A. Mammela, "Performance improvement with predictive channel selection for cognitive radios," in Proc. CogART, 2008. 